\title{
Some Topological Invariants of Graphs Associated with the Group of Symmetries
}

\author{
Chang-Cheng Wei, ${ }^{1}$ Muhammad Salman $\mathbb{D}^{2},{ }^{2}$ Usman Ali $\mathbb{D}^{\mathrm{D}},{ }^{3,4}$ Masood Ur Rehman (D), \\ Muhammad Aqeel Ahmad Khan, ${ }^{7}$ Muhammad Hasanain Chaudary, ${ }^{8}$ \\ and Farooq Ahmad $\oplus^{8}$ \\ ${ }^{1}$ Department of Mathematics and Computer Science, Anhui TongLing University, Tongling 244061, China \\ ${ }^{2}$ Department of Mathematics, The Islamia University of Bahawalpur, Bahawalpur, Pakistan \\ ${ }^{3}$ Centre for Advanced Studies in Pure and Applied Mathematics (CASPAM), Bahauddin Zakaryia University, Multan, Pakistan \\ ${ }^{4}$ Institut de Mathématiques de Jussieu-Paris Rive Gauche, Paris, France \\ ${ }^{5}$ Department of Mathematics, Abbottabad University of Science and Technology, Abbottabad, Pakistan \\ ${ }^{6}$ Department of Basic Sciences, Balochistan University of Engineering and Technology, Khuzdar, Balochistan, Pakistan \\ ${ }^{7}$ Department of Mathematics, COMSATS University Islamabad, Lahore Campus, Lahore, Pakistan \\ ${ }^{8}$ Department of Computer Science, COMSATS University Islamabad, Lahore Campus, Lahore, Pakistan
}

Correspondence should be addressed to Usman Ali; uali@bzu.edu.pk and Masood Ur Rehman; masood@mail.ustc.edu.cn

Received 21 August 2019; Accepted 9 October 2019; Published 9 March 2020

Academic Editor: Teodorico C. Ramalho

Copyright () 2020 Chang-Cheng Wei et al. This is an open access article distributed under the Creative Commons Attribution License, which permits unrestricted use, distribution, and reproduction in any medium, provided the original work is properly cited.

A topological index is a quantity that is somehow calculated from a graph (molecular structure), which reflects relevant structural features of the underlying molecule. It is, in fact, a numerical value associated with the chemical constitution for the correlation of chemical structures with various physical properties, chemical reactivity, or biological activity. A large number of properties like physicochemical properties, thermodynamic properties, chemical activity, and biological activity can be determined with the help of various topological indices such as atom-bond connectivity indices, Randić index, and geometric arithmetic indices. In this paper, we investigate topological properties of two graphs (commuting and noncommuting) associated with an algebraic structure by determining their Randić index, geometric arithmetic indices, atomic bond connectivity indices, harmonic index, Wiener index, reciprocal complementary Wiener index, Schultz molecular topological index, and Harary index.

\section{Introduction}

In quantitative structure-activity relationship (QSAR)/ quantitative structure-property relationship (QSPR) study, physicochemical properties and topological indices such as Randić index, atom-bond connectivity (ABC) index, and geometric-arithmetic (GA) index are used to predict the bioactivity of chemical compounds. A topological index is actually designed by transforming a chemical structure into a numerical number. It correlates certain physicochemical properties such as boiling point, stability, and strain energy of chemical compounds of a molecular structure (graph). It is a numeric quantity associated with a chemical structure (graph), which characterizes the topology of the structure and is invariant under a structure-preserving mapping [1]. In 1947, Wiener [2] introduced the concept of (distancebased) topological index while working on the boiling point of paraffin. He named this index as the path number. Later on, the path number was renamed as the Wiener index [2], and then, the theory of topological indices started. Nowadays, a number of distance-based and degree-based topological indices have been introduced and computed (see for example [3-15], and the references therein).

We consider simple and connected graph (chemical structure) $G$ with vertex set $V(G)$ and edge set $E(G)$. We denote the two adjacent vertices $u$ and $v$ in $G$ as $u \sim v$ and nonadjacent vertices as $u \nsim v$. The number $d_{v}$ denotes the degree of a vertex $v \in V(G)$ and $S_{v}=\sum_{u \in N(v)} d_{u}$ is the 
TABLE 1: List of under consideration topological indices.

\begin{tabular}{|c|c|c|}
\hline Name of the index & Notation & Formula \\
\hline Wiener index [2] & $W(G)$ & $\sum_{\{u, v\} \in V(G)} d(u, v)$ \\
\hline Reciprocal complementary Wiener index [21] & $\operatorname{RCW}(G)$ & $\sum_{\{u, v\} \in V(G)} 1 /(D(G)+1-d(u, v))$ \\
\hline Schultz molecular topological index [22] & $\operatorname{MTI}(G)$ & $\sum_{\{u, v\} \in V(G)}\left(d_{u}+d_{v}\right) d(u, v)+\sum_{v \in V(G)} d^{2 / v}$ \\
\hline Harary index $[23,24]$ & $H(G)$ & $\sum_{\{u \neq v\} \in V(G)} 1 /(d(u, v))$ \\
\hline Randić index [10] & $R_{-1 / 2}(G)$ & $\sum_{u \sim v} 1 /\left(\sqrt{d_{u} \times d_{v}}\right)$ \\
\hline General Randić index $[11,12]$ & $R_{\alpha}(G)$ & $\sum_{u \sim v}\left(d_{u} \times d_{v}\right)^{\alpha}$ \\
\hline Geometric arithmetic (GA) index [13] & $\mathrm{GA}(G)$ & $\mathrm{s}$ \\
\hline Fifth version of GA index [4] & $\mathrm{GA}_{5}(G)$ & $\sum_{u \sim v}\left(2 \sqrt{S_{u} \times S_{v}}\right) /\left(S_{u}+S_{v}\right)$ \\
\hline Atomic bond connectivity (ABC) index [14] & $\operatorname{ABC}(G)$ & $\sum_{u \sim v} \sqrt{\left(d_{u}+d_{v}-2\right) /\left(d_{u} \times d_{v}\right)}$ \\
\hline Fourth version of $\mathrm{ABC}$ index [5] & $\mathrm{ABC}_{4}(G)$ & $\sum_{u \sim v} \sqrt{\left(S_{u}+S_{v}-2\right) /\left(S_{u} \times S_{v}\right)}$ \\
\hline Harmonic index [15] & $H_{r}(G)$ & $\sum_{u \sim v}^{u} 2 /\left(d_{u}+d_{v}\right)$ \\
\hline
\end{tabular}

All the notations used in formulas are defined in Section 1.

degrees sum of $v$, where $N(v)=\{u \in V(G) \mid u \sim v$ in $G\}$ is the neighborhood of $v$. The number $d(u, v)$ denotes the length of a geodesic between $u$ and $v$ in $G$ and is called the distance between $u$ and $v$. The eccentricity of a vertex $v$ in $G$, denoted by $\operatorname{ecc}(v)$, is the maximum distance between $v$ and any other vertex of $G$. The minimum eccentricity amongst the vertices of $G$ is called the radius of $G$, denoted by $\operatorname{rad}(G)$. The diameter of a graph $G$ is the maximum eccentricity in $G$, denoted by $D(G)$. A vertex $v$ in $G$ is said to be a central vertex if ecc $(v)=\operatorname{rad}(G)$, and the subgraph of $G$ induced by central vertices of $G$ is called the center of $G$. A vertex $v$ in a graph $G$ is called a peripheral vertex if $\operatorname{ecc}(v)=D(G)$, and the subgraph of $G$ induced by peripheral vertices is called the periphery of $G$. The sum of two graphs $G_{1}$ and $G_{2}$, denoted by $G_{1}+G_{2}$, is a graph with vertex set $V\left(G_{1}\right) \cup V\left(G_{2}\right)$ and an edge set $E\left(G_{1}\right) \cup E\left(G_{2}\right) \cup\left\{u \sim v: u \in V\left(G_{1}\right) \wedge v \in V\left(G_{2}\right)\right\}$.

Let $\Gamma$ be a group. The set $\zeta(\Gamma)=\{x: x \in \Gamma \wedge x y=y x \forall y \in \Gamma\}$ is called the center of the group $\Gamma$. Then, commuting and noncommuting graphs of $\Gamma$ are defined as follows:

(i) The commuting graph of a nonabelian group $\Gamma$ is denoted by $\Gamma_{G}=C(\Gamma, \Omega)$ with vertex set $\Omega \subseteq \Gamma$. For two distinct elements $x, y \in \Omega, x \sim y$ in $\Gamma_{G}(x$ and $y$ form an edge in $\Gamma_{G}$ ) if and only if $x y=y x$ in $\Gamma$. The concept of commuting graphs on noncentral elements of a group has been studied by various researchers (see $[16,17]$ ).

(ii) The noncommuting graph of a nonabelian group $G_{\Gamma}$ is a graph with vertex set $V\left(G_{1}\right) \cup V\left(G_{2}\right)$, and two distinct vertices $u$ and $v$ in $G_{\Gamma}$ form an edge if $u v \neq v u$ in $\Gamma$. The study of noncommuting graphs of groups was initiated in 1975 by Neumann [18] who posed the problem regarding the clique number of a noncommuting graph. Noncommuting graphs on noncentral elements of a group have also been studied by various other researchers $[19,20]$.

The following useful property for a noncommuting graph was proposed in [19].

Proposition 1 (see [19]). For any nonabelian group $\Gamma$, $D\left(G_{\Gamma}\right)=2$.
A graph $G$ is said to be self-centered if $\operatorname{rad}(G)=D(G)$. Since ecc $(v) \leq 2$ for every $v \in G_{\Gamma}$, so we have the following straightforward proposition:

Proposition 2. A noncommuting graph $G_{\Gamma}$ of any nonabelian finite group $\Gamma$ is self-centered if for each $v \in G_{\Gamma}$, $\operatorname{ecc}(v)=2$. Otherwise, it is the sum of the center and the periphery of $G_{\Gamma}$.

This paper aimed at investigating all the topological properties (listed in Table 1) of commuting and noncommuting graphs associated with the group of symmetries. The rest of the paper consists of five sections. In the next section, we illustrate the group of symmetries and associate commuting and noncommuting graphs to this group. In Section 3, some useful constructions to investigate our main results of Sections 4 and 5 are provided.

\section{Group of Symmetries and Associated Graphs}

Group of symmetries finds its remarkable use in the theory of electron structures and molecular vibrations. Due to their significant employment in chemical structures, in the context of topological indices, we consider the group of symmetries of a regular polygon (also called a regular $n$-gon for $n \geq 2$ ) in this paper. A regular $n$-gon is a geometrical figure all of whose sides have the same length and all the angles are of equal measurement. Each internal angle of a $n$-gon is of $\pi-(2 \pi) /(n)$ radian. The group of symmetries of a $n$-gon consists of $2 n$ elements, which are $n$ rotations $\left(r_{0}=e, r_{1}, r_{2}, \ldots, r_{n-1}\right.$ about its center through an angle of $(2 k \pi) / n$ radian, where $k=0,1, \ldots, n-1$, either all clockwise or all anticlockwise) and $n$ reflections (for even $n$, the reflections through a line joining the midpoints of the opposite sides or through a line joining two opposite vertices, and for odd $n$, the reflections through those lines which join a vertex with the midpoint of the opposite side). The group of symmetries is denoted by $D_{n}$ and is called the dihedral group of order $2 n$. If we denote a rotation by " $a$ " and a reflection by " $b$," then $2 n$ elements of $D_{n}$ are $a, a^{2}, \ldots, a^{n-1}, a^{n}=e$ and $b, a b, a^{2} b, \ldots, a^{n-1} b$, where $e$ is the identical rotation. The general representation of $D_{n}$ is given by 
TABLE 2: Vertex partition of $\Gamma_{G}$ for each vertex $v \in V\left(\Gamma_{G}\right)$.

\begin{tabular}{lccccc}
\hline$n$ is & $d_{v}$ & $D\left(v \mid \Gamma_{G}\right)$ & $D_{s}\left(v \mid \Gamma_{G}\right)$ & $D_{r}\left(v \mid \Gamma_{G}\right)$ & Number of vertices \\
\hline Odd & $n-1$ & $3 n-1$ & $(1 / 2)(3 n-1)$ & $(1 / 2)(3 n-2)$ & $n-1$ \\
Odd & $2 n-1$ & $2 n-1$ & $(1 / 2)(2 n-1)$ & $2 n-1$ & $n$ \\
Odd & 1 & $4 n-3$ & $(1 / 2)(4 n-3)$ & $(1 / 2)(3 n-2)$ & $n$ \\
Even & $n-1$ & $3 n-1$ & $(1 / 2)(3 n-1)$ & $2 n-1$ & $n$ \\
Even & $2 n-1$ & $2 n-1$ & $(1 / 2)(2 n-1)$ & $n+1$ & 2 \\
Even & 3 & $4 n-5$ & $(1 / 2)(4 n-5)$ & \\
\hline
\end{tabular}

$$
D_{n}=\left\langle a, b \mid a^{n}=b^{2}=e, a b=b a^{-1}\right\rangle,
$$

with the center

$$
\zeta\left(D_{n}\right)= \begin{cases}\{e\}, & \text { when } n \text { is odd } \\ \left\{e, a^{n / 2}\right\}, & \text { when } n \text { is even. }\end{cases}
$$

Let $\Omega_{1}=\left\{e, a, a^{2}, \ldots, a^{n-1}\right\}, \Omega_{2}=\left\{b, a b, a^{2} b, \ldots, a^{n-1} b\right\}$, and $\Omega_{3}=\Omega_{1}-\zeta\left(D_{n}\right)$. Then $\left|\Omega_{1}\right|=n=\left|\Omega_{2}\right|$ and

$$
\left|\Omega_{3}\right|= \begin{cases}n-1, & \text { when } n \text { is odd, } \\ n-2, & \text { when } n \text { is even. }\end{cases}
$$

In the case of even value of $n \geq 4$, we partitioned $\Omega_{2}$ into $n / 2$ two element subsets $\Omega_{2}^{i}=\left\{a^{i} b, a^{((n / 2)+i)} b\right\}$, $0 \leq i \leq(n / 2)-1$, so that $\Omega_{2}=\cap_{i=0}^{(n / 2)-1} \Omega_{2}^{i}$.

Remark 1. In the dihedral group $D_{n}$, we have

(i) $x y=y x$ for all $x, y \in D_{2}$

(ii) $a^{i} b=b a^{n-i}$ for $i=1,2, \ldots, n-1$

(iii) For odd values of $n \geq 3, x y \neq y x$ for distinct $x, y \in \Omega_{2}$

(iv) For even values of $n \geq 4$, and for any distinct $x, y \in \Omega_{2}, \quad x y=y x$ if and only if $x, y \in \Omega_{2}^{i}$, $0 \leq i \leq(n / 2)-1$

(v) For any distinct $x, y \in \Omega_{3}, x y=y x$

(vi) For each pair $(x, y) \in \Omega_{2} \times \Omega_{3}, x y \neq y x$

According to Remark 1, the commuting graph on $D_{n}$ is defined in the following result.

Proposition 3 (see [16]). For all $n \geq 3$, let $\Gamma_{G}=\mathscr{C}\left(D_{n}, D_{n}\right)$ be a commuting graph on $D_{n}$, then

$$
\Gamma_{G}= \begin{cases}K_{1}+\left(K_{\left|\Omega_{3}\right|} \cup N_{\left|\Omega_{1}\right|}\right), & \text { when } n \text { is odd, } \\ K_{2}+\left(K_{\left|\Omega_{3}\right|} \cup \frac{n}{2} K_{2}\right), & \text { when } n \text { is even. }\end{cases}
$$

Here, $K_{1}$ is the trivial graph, $K_{p}$ is a complete graph on $p$ vertices, $N_{t}$ is a null (empty) graph on $t$ vertices, and $(n / 2) K_{2}$ is the union of $(n / 2)$ copies of $K_{2}$.

Let $\Gamma=D_{n}, n \geq 3$, and $G_{\Gamma}$ be the corresponding noncommuting graph. Then, according to Remark 1, we have the following points:

When $n \geq 3$ is odd, then
(1) For $u, v \in V\left(G_{\Gamma}\right), u \sim v$ whenever $u, v \in \Omega_{2}$.

(2) For $u, v \in V\left(G_{\Gamma}\right), u \sim v$ whenever $u, v \in \Omega_{3}$.

(3) For $u, v \in V\left(G_{\Gamma}\right), u \sim v$ whenever $u \in \Omega_{2}$ and $v \in \Omega_{3}$.

(4) In $G_{\Gamma}$, it can be seen that $\operatorname{ecc}(v)=1$ for all $v \in \Omega_{2}$, and $\operatorname{ecc}(v)=2$ for all $v \in \Omega_{3}$. It follows that $\Omega_{2}$ induces the center of $G_{\Gamma}$, which is a complete graph $K_{\left|\Omega_{2}\right|}$ on $\left|\Omega_{2}\right|$ vertices, and $\Omega_{3}$ induces the periphery of $G_{\Gamma}$, which is a null graph $N_{\left|\Omega_{3}\right|}$ on $\left|\Omega_{3}\right|$ vertices.

When $n \geq 4$ is even, then

(1) For $u, v \in V\left(G_{\Gamma}\right), u+v$ whenever $u, v \in \Omega_{2}^{i}$ for any $0 \leq i \leq(n / 2)-1$.

(2) For $u, v \in V\left(G_{\Gamma}\right), u \nsim v$ whenever $u, v \in \Omega_{3}$.

(3) For $u, v \in V\left(G_{\Gamma}\right), u \sim v$ whenever $u \in \Omega_{2}$ and $v \in \Omega_{3}$.

(4) For $u, v \in V\left(G_{\Gamma}\right), u \sim v$ whenever $u \in \Omega_{2}^{i}$ and $v \in \Omega_{2}^{j}$ with $0 \leq i, j \leq(n / 2)-1$ and $i \neq j$.

(5) In $G_{\Gamma}$, it can be seen that $\operatorname{ecc}(v)=2$ for all $v \in \Omega_{2} \cup \Omega_{3}$. It follows that $G_{\Gamma}$ is a self-centered graph, which is a complete multipartite graph $K_{2,2, \ldots, 2},\left|\Omega_{3}\right| \quad$ with $n / 2 \quad$ partite sets $\Omega_{2}^{i}, 0 \leq i \leq(n / 2)-1$, and one partite set $\Omega_{3}$.

Hence, by Proposition 2, we deduce the following result.

Proposition 4. For $n \geq 3$, let $\Gamma=D_{n}$. Then, the noncommuting graph $G_{\Gamma}$ of $D_{n}$ is given by

$$
G_{\Gamma}= \begin{cases}K_{\Omega_{2}}+N_{\Omega_{3}}, & \text { when } n \text { is odd, } \\ \underbrace{K_{2,2, \ldots, 2},\left|\Omega_{3}\right|}_{(n / 2) \text {-times }}, & \text { when } n \text { is even. }\end{cases}
$$

\section{Construction of Vertex and Edge Partitions}

First we define some useful parameters, which support in the investigation of some predefined (in Table 1) topological indices. For any vertex $v$ of $G$, these parameters are defined as follows:

(i) The distance number of $v$ in $G$ is $D(v \mid G)=\sum_{u \in V(G)} d(u, v)$

(ii) The sum distance number of $v$ in $G$ is $D_{s}(v \mid G)=\sum_{u \in V(G)-\{v\}} 1 /(D(G)+1-d(u, v))$

(iii) The reciprocal distance number of $v$ in $G$ is $D_{r}(v \mid G)=\sum_{u \in V(G)} 1 /(d(u, v))$

According to these parameters, the distance-based topological indices, listed in Table 1, become 
TABLE 3: Edge partition of $\Gamma_{G}$ for each edge $u \sim v \in E\left(\Gamma_{G}\right)$.

\begin{tabular}{lccc}
\hline$n$ is & $\left(d_{u}, d_{v}\right)$ type edges & $\left(S_{u}, S_{v}\right)$ type edges & Number of edges \\
\hline Odd & $(n-1, n-1)$ & $\left(n^{2}-n+1, n^{2}-n+1\right)$ & $((n-1)(n-2)) / 2$ \\
Odd & $(n-1,2 n-1)$ & $\left(n^{2}-n+1, n^{2}-n+1\right)$ & $n-1$ \\
Odd & $(1,2 n-1)$ & $\left(2 n-n, n^{2}-n+1\right)$ & $n$ \\
Even & $(n-1, n-1)$ & $\left(n^{2}+1, n^{2}+1\right)$ & $((n-2)(n-3)) / 2$ \\
Even & $(n-1,2 n-1)$ & $\left(n^{2}+1,(n+1)^{2}\right)$ & $2(n-2)$ \\
Even & $(2 n-1,2 n-1)$ & $\left((n+1)^{2},(n+1)^{2}\right)$ & 1 \\
Even & $(2 n-1,3)$ & $\left((n+1)^{2}, 4 n+1\right)$ & $2 n$ \\
Even & $(3,3)$ & $(4 n+1,4 n+1)$ & $n / 2$ \\
\hline
\end{tabular}

Note: $\left(d_{u}, d_{v}\right)$ denotes the type of edge $u \sim v$ according to degrees of the end vertices, and $\left(S_{u}, S_{v}\right)$ denotes the type of edge $u \sim v$ according to degrees sum of the end vertices.

TABLE 4: Vertex partition of $G_{\Gamma}$ for each vertex $v \in V\left(G_{\Gamma}\right)$.

\begin{tabular}{lcccccc}
\hline$n$ is & $d_{v}$ & ecc $(v)$ & $D\left(v \mid G_{\Gamma}\right)$ & $D_{s}\left(v \mid G_{\Gamma}\right)$ & $D_{r}\left(v \mid G_{\Gamma}\right)$ & Number of vertices \\
\hline Odd & $2 n-2$ & 1 & $2 n-2$ & $n-1$ & $2 n-2$ & $n$ \\
Odd & $n$ & 2 & $3 n-4$ & $(1 / 2)(3 n-4)$ & $(1 / 2)(3 n-1)$ & $n-1$ \\
Even & $2 n-2$ & 2 & $2 n-2$ & $n-1$ & $(1 / 2)(4 n-7)$ & $n$ \\
Even & $n$ & 2 & $3 n-6$ & $(3 / 2)(n-2)$ & $(3 / 2)(n-1)$ & $n-2$ \\
\hline
\end{tabular}

TABLE 5: Edge partition of $G_{\Gamma}$ for each edge $u \sim v \in E\left(G_{\Gamma}\right)$.

\begin{tabular}{lccc}
\hline$n$ is & $\left(d_{u}, d_{v}\right)$ type edges & $\left(S_{u}, S_{v}\right)$ type edges & Number of edges \\
\hline Odd & $(n, 2 n-2)$ & $(2 n(n-1),(n-1)(3 n-2))$ & $n(n-1)$ \\
Odd & $(2 n-2,2 n-2)$ & $((n-1)(3 n-2),(n-1)(3 n-2))$ & $(n(n-1)) / 2$ \\
Even & $(n, 2 n-4)$ & $(2 n(n-2),(n-2)(3 n-4))$ & $n(n-2)$ \\
Even & $(2 n-4,2 n-4)$ & $((n-2)(3 n-4),(n-2)(3 n-4))$ & $(n(n-2)) / 2$ \\
\hline
\end{tabular}

Note: $\left(d_{u}, d_{v}\right)$ denotes the type of edge $u \sim v$ according to degrees of the end vertices, and $\left(S_{u}, S_{v}\right)$ denotes the type of edge $u \sim v$ according to degrees sum of the end vertices.

$$
\begin{aligned}
W(G) & =\frac{1}{2} \sum_{v \in V(G)} D(v \mid G), \\
\operatorname{RCW}(G) & =\frac{|G|}{D(G)+1}+\frac{1}{2} \sum_{v \in V(G)} D_{s}(v \mid G), \\
\operatorname{MTI}(G) & =\sum_{v \in V(G)}(d(v))^{2}+\sum_{v \in V(G)} d(v) D(v \mid G), \\
H(G) & =\frac{1}{2} \sum_{v \in V(G)} D_{r}(v \mid G) .
\end{aligned}
$$

Let $\Gamma_{G}$ be a commuting graph of the dihedral group $D_{n}$. In $\Gamma_{G}$, there are $2 n$ vertices. The number of edges in $\Gamma_{G}$ is $(n(n+1)) / 2$ when $n$ is odd and is $(n(n+4)) / 2$ when $n$ is even. Based on the degree, distance number, sum distance number, and reciprocal distance number of each vertex of $\Gamma_{G}$, the useful vertex partition is given in Table 2. Based on degrees and degrees sum of the end vertices of each edge of $\Gamma_{G}$, the useful edge partition is given in Table 3.

Let $G_{\Gamma}$ be a non-commuting graph of the dihedral group $D_{n}$. In $G_{\Gamma}$,
(1) There are $2 n-1$ vertices and $(3 / 2) n(n-1)$ edges when $n$ is odd

(2) There are $2 n-2$ vertices and $(3 / 2) n(n-2)$ edges when $n$ is even

Based on the degree, eccentricity, distance number, sum distance number, and reciprocal distance number of each vertex of $G_{\Gamma}$, the useful vertex partition is given in Table 4. Based on degrees and degrees sum of the end vertices of each edge of $G_{\Gamma}$, the useful edge partition is given in Table 5.

\section{Topological Properties of Commuting Graph $\Gamma_{G}$}

In this section, we compute the Wiener, reciprocal complementary Wiener, MTI, Harary general Randić, ABC, $\mathrm{ABC}_{4}, \mathrm{GA}, \mathrm{GA}_{5}$, and harmonic indices of $\Gamma_{G}$. Throughout this section, in each of the two-row equation arrays, the first row corresponds to odd values of $n$, while the second corresponds to even values of $n$.

Theorem 1. For $n \geq 3$, let $\Gamma_{G}$ be a commuting graph on $D_{n}$, then 


$$
W\left(\Gamma_{G}\right)= \begin{cases}\frac{n}{2}(7 n-5), & \text { when } n \text { is odd } \\ \frac{n}{2}(7 n-8), & \text { when } n \text { is even }\end{cases}
$$

Proof. Using the vertex partition, given in Table 2, in formula (6) of the Wiener index, we have

$$
W\left(\Gamma_{G}\right)= \begin{cases}\frac{(n-1)(3 n-1)+(2 n-1)+n(4 n-3)}{2}, & \text { when } n \text { is odd } \\ \frac{(n-2)(3 n-1)+2(2 n-1)+n(4 n-5)}{2}, & \text { when } n \text { is even. }\end{cases}
$$

Now, the required Wiener index can be obtained after some simplifications.

Theorem 2. For $n \geq 3$, let $\Gamma_{G}$ be a commuting graph on $D_{n}$, then

$$
\operatorname{RCW}\left(\Gamma_{G}\right)= \begin{cases}\frac{7 n}{12}(3 n-1), & \text { when } n \text { is odd, } \\ \frac{n}{12}(21 n-16), & \text { when } n \text { is even. }\end{cases}
$$

Proof. Since the diameter of $G_{\Gamma}$ is 2 , so by using the vertex partition, given in Table 4, in formula (7) of the reciprocal complimentary Wiener index, we have

$$
\operatorname{RCW}\left(\Gamma_{G}\right)= \begin{cases}\frac{2 n}{3}+\frac{1}{2}\left(\frac{n}{2}(4 n-3)+\frac{1}{2}(2 n-1)+\frac{1}{2}(n-1)(3 n-1)\right), & \text { when } n \text { is odd } \\ \frac{2 n}{3}+\frac{1}{2}\left(\frac{n}{2}(4 n-5)+(2 n-1)+\frac{1}{2}(n-2)(3 n-1)\right), & \text { when } n \text { is even. }\end{cases}
$$

Now, the required index can be easily found by performing some simplifications.

Theorem 3. For $n \geq 3$, let $\Gamma_{G}$ be a commuting graph on $D_{n}$, then

$$
\operatorname{MTI}\left(\Gamma_{G}\right)= \begin{cases}2 n(2 n-1)(n+1), & \text { when } n \text { is odd, } \\ 2 n(2 n-1)(n+4), & \text { when } n \text { is even. }\end{cases}
$$

Proof. By applying formula (8) of the Schultz molecular topological index using the vertex partition, given in Table 2, we have when $n$ is odd

$$
\begin{aligned}
\operatorname{MTI}\left(\Gamma_{G}\right)= & (n-1)^{3}+(2 n-1)^{2}+n+(n-1)^{2}(3 n-1) \\
& +(2 n-1)^{2}+n(4 n-3) \\
= & 2 n(2 n-1)(n+1),
\end{aligned}
$$

and when $n$ is even

$$
\begin{aligned}
\operatorname{MTI}\left(\Gamma_{G}\right)= & (n-2)(n-1)^{2}+2(2 n-1)^{2}+n(3)^{2} \\
& +(n-2)(n-1)(3 n-1)+2(2 n-1)^{2} \\
& +3 n(4 n-5)=2 n(2 n-1)(n+4) .
\end{aligned}
$$

Theorem 4. For $n \geq 3$, let $\Gamma_{G}$ be a commuting graph on $D_{n}$, then

$$
H\left(\Gamma_{G}\right)= \begin{cases}\frac{n}{4}(5 n-1), & \text { when } n \text { is odd } \\ \frac{n}{4}(5 n+2), & \text { when } n \text { is even }\end{cases}
$$

Proof. Using the vertex partitions, given in Table 2, in formula (9) of the Harary index, we have 


$$
H\left(\Gamma_{G}\right)= \begin{cases}\frac{1}{2}\left(\frac{(n-1)(3 n-2)}{2}+2 n-1+n^{2}\right), & \text { when } n \text { is odd, } \\ \frac{1}{2}\left(\frac{(n-2)(3 n-2)}{2}+2(2 n-1)+n(n+1)\right), & \text { when } n \text { is even. }\end{cases}
$$

Some easy simplifications yield the required Harary index.
Theorem 5. For $n \geq 3$, let $\Gamma_{G}$ be a commuting graph of $\Gamma=D_{n}$. Then, for odd values of $n$,

$$
R_{\alpha}\left(\Gamma_{G}\right)= \begin{cases}\frac{n}{2}\left(n^{3}-n^{2}+3 n-1\right), & \text { for } \alpha=1 \\ \frac{n(4 n-5)}{(2 n-2)(2 n-1)}, & \text { for } \alpha=-1 \\ \frac{(n-1)^{2}(n-2)+2(n-1) \sqrt{(n-1)(2 n-1)}+2 n \sqrt{2 n-1}}{2}, & \text { for } \alpha=\frac{1}{2} \\ \frac{n-2 \sqrt{2 n-1}+2 \sqrt{n-1}+2 n}{2 \sqrt{2 n-1},} & \text { for } \alpha=-\frac{1}{2}\end{cases}
$$

and for even values of $n$,

$$
R_{\alpha}\left(\Gamma_{G}\right)= \begin{cases}\frac{1}{2}\left((n-1)^{2}\left(n^{2}-5 n+6\right)+2(2 n-1)\left(2 n^{2}+2 n+3\right)+9 n\right), & \text { for } \alpha=1, \\ \frac{9(2 n-1)\left(2 n^{3}-7 n^{2}+5 n+2\right)^{2}\left(4 n^{3}+20 n^{2}-11 n+18\right)}{18(n-1)^{2}(2 n-1)^{2}}, & \text { for } \alpha=-1, \\ \frac{(n-1)(n-2)(n-3)+7 n-2+4 \sqrt{2 n-1}((n-2) \sqrt{n-1}+n \sqrt{3})}{2}, & \text { for } \alpha=\frac{1}{2}, \\ \frac{2 n(n-1)(2 n-7)+3(5 n-4)}{3(n-1)(2 n-1)}+\frac{2(n-2) \sqrt{3}+2 n \sqrt{n-1}}{\sqrt{3(2 n-1)(n-1)}}, & \text { for } \alpha=-\frac{1}{2} .\end{cases}
$$

Proof. Using the edge partition, given in Table 3, in the formula of general Randić index $R_{\alpha}$ for $\alpha=1,-1,(1 / 2),-(1 / 2)$, we have 


$$
\begin{aligned}
& R_{1}\left(\Gamma_{G}\right)=\left\{\begin{array}{l}
\frac{(n-1)^{3}(n-2)}{2}+(n-1)^{2}(2 n-1)+n(2 n-1), \\
\frac{(n-2)(n-3)(n-1)^{2}}{2}+2(n-2)(n-1)(2 n-1)+(2 n-1)^{2}+6 n(2 n-1)+\frac{9 n}{2},
\end{array}\right. \\
& R_{-1}\left(\Gamma_{G}\right)=\left\{\begin{array}{l}
\frac{(n-2)}{2(n-1)}+\frac{1}{2 n-1}+\frac{n}{2 n-1}, \\
\frac{(n-2)(n-3)}{2(n-1)^{2}}+\frac{2(n-2)}{(n-1)(2 n-1)}+\frac{1}{(2 n-1)^{2}}+\frac{2 n}{3(2 n-1)}+\frac{n}{18},
\end{array}\right. \\
& R_{1 / 2}\left(\Gamma_{G}\right)=\left\{\begin{array}{l}
\frac{(n-1)(n-2) \sqrt{(n-1)^{2}}}{2}+(n-1) \sqrt{(n-1)(2 n-1)}+n \sqrt{2 n-1}, \\
\frac{(n-2)(n-3)(n-1)}{2}+2(n-2) \sqrt{(n-1)(2 n-1)}+2 n-1+2 n \sqrt{3(2 n-1)}+\frac{3 n}{2},
\end{array}\right. \\
& R_{-(1 / 2)}\left(\Gamma_{G}\right)=\left\{\begin{array}{l}
\frac{(n-1)(n-2)}{2 \sqrt{(n-1)(n-1)}}+\frac{(n-1)}{\sqrt{(n-1)(2 n-1)}}+\frac{n}{\sqrt{2 n-1}}, \\
\frac{(n-2)(n-3)}{2(n-1)}+\frac{2(n-2)}{\sqrt{(n-1)(2 n-1)}}+\frac{1}{2 n-1}+\frac{2 n}{\sqrt{3(2 n-1)}}+\frac{n}{6} .
\end{array}\right.
\end{aligned}
$$

After a minor simplification, we get our required result.
Theorem 6. For $n \geq 3$, let $\Gamma_{G}$ be a commuting graph of $\Gamma=D_{n}$, then

$$
\begin{aligned}
& \mathrm{GA}\left(\Gamma_{G}\right)=\left\{\begin{array}{l}
\frac{(n-1)(n-2)}{2}+\frac{2(n-1) \sqrt{(n-1)(2 n-1)}}{3 n-2}+\sqrt{2 n-1}, \\
\frac{n^{2}-4 n+8}{2}+\frac{4\left(n^{2}-n-2\right) \sqrt{2 n^{2}-3 n+1}+2 n(3 n-2) \sqrt{3(2 n-1)}}{(n+1)(3 n-2)},
\end{array}\right. \\
& \mathrm{GA}_{5}\left(\Gamma_{G}\right)=\left\{\begin{array}{l}
\frac{n\left(n^{2}-1\right)+4 \sqrt{n^{3}+(n-1)^{3}}}{2(n+1)}, \\
\frac{n^{2}-4 n+8}{2}+\frac{2(n-2)(n+1) \sqrt{n^{2}+1}}{n^{2}+n+1}+\frac{4 n(n+1) \sqrt{4 n+1}}{n^{2}+6 n+2} .
\end{array}\right.
\end{aligned}
$$

Proof. Applying formulas of the geometric arithmetic index and its fifth version, using the edge partition given in Table 3 , we have 


$$
\begin{aligned}
& \mathrm{GA}\left(\Gamma_{G}\right)=\left\{\begin{array}{l}
\frac{2(n-1)^{2}(n-2)}{2(2 n-2)}+\frac{2(n-1) \sqrt{(n-1)(2 n-1)}}{3 n-2}+\frac{2 n \sqrt{2 n-1}}{2 n}, \\
\frac{(n-2)(n-3)}{2}+\frac{4(n-2) \sqrt{(n-1)(2 n-1)}}{3 n-2}+1+\frac{2 n \sqrt{3(2 n-1)}}{n+1}+\frac{n}{2},
\end{array}\right. \\
& \mathrm{GA}_{5}\left(\Gamma_{G}\right)=\left\{\begin{array}{l}
\frac{2 n(n-1) \sqrt{\left(n^{2}-n+1\right)^{2}}}{4\left(n^{2}-n+1\right)}+\frac{2 n \sqrt{\left(n^{2}-n+1\right)(2 n-1)}}{n(n+1)}, \\
\frac{(n-2)(n-3)}{2}+\frac{4(n-2) \sqrt{\left(n^{2}+1\right)(n+1)^{2}}}{2 n^{2}+2 n+2}+1+\frac{4 n \sqrt{(n+1)^{2}(4 n+1)}}{n^{2}+6 n+2}+\frac{n}{2} .
\end{array}\right.
\end{aligned}
$$

The required values of the geometric arithmetic index and its fifth version can be obtained after some simplifications.
Theorem 7. For $n \geq 3$, let $\Gamma_{G}$ be a commuting graph of $\Gamma=D_{n}$, then

$$
\begin{aligned}
& \operatorname{ABC}\left(\Gamma_{G}\right)=\left\{\begin{array}{l}
\frac{(n-2) \sqrt{(n-2)(2 n-1)}+\sqrt{2(n-1)(3 n-4)}+2 n \sqrt{n-1}}{\sqrt{2(2 n-1)}}, \\
\frac{(n-2)(n-3) \sqrt{2(n-2)}}{2(n-1)}+\frac{2 \sqrt{n-1}}{(2 n-1)}+\frac{n}{3}+\frac{2 n \sqrt{2 n(n-1)}+2(n-2) \sqrt{3(3 n-4)}}{\sqrt{3(n-1)(2 n-1)}},
\end{array}\right. \\
& \mathrm{ABC}_{4}\left(\Gamma_{G}\right)=\left\{\begin{array}{l}
\frac{n(n-1) \sqrt{2 n(n-1)}}{2\left(n^{2}-n+1\right)}+n \sqrt{\frac{n^{2}+n-2}{(2 n-1)\left(n^{2}-n+1\right)}}, \\
\frac{n(n-2)(n-3) \sqrt{2}}{2\left(n^{2}+1\right)}+\frac{2(n-2)}{n+1} \sqrt{\frac{2 n(n+1)}{n^{2}+1}}+\frac{\sqrt{2 n(n+2)}}{(n+1)^{2}}+\frac{2 n}{n+1} \sqrt{\frac{n(n+6)}{4 n+1}+\frac{n \sqrt{2 n}}{4 n+1}} .
\end{array}\right.
\end{aligned}
$$

Proof. By using the edge partition, given in Table 3 , in formulas of $\mathrm{ABC}$ and $\mathrm{ABC}_{4}$ indices, we have

$$
\operatorname{ABC}\left(\Gamma_{G}\right)=\left\{\begin{array}{l}
\frac{(n-2) \sqrt{2(n-2)}}{2}+(n-1) \sqrt{\frac{3 n-4}{(n-1)(2 n-1)}}+n \sqrt{\frac{2(n-1)}{2 n-1}} \\
\frac{(n-2)(n-3) \sqrt{2(n-2)}}{2(n-1)}+2(n-2) \sqrt{\frac{3 n-4}{(n-1)(2 n-1)}}+\frac{2 \sqrt{n-1}}{2 n-1}+\frac{n}{3}+2 n \sqrt{\frac{2 n}{3(2 n-1)}}
\end{array}\right.
$$

Also, for odd values of $n$, we have

$$
\begin{aligned}
\operatorname{ABC}_{4}\left(\Gamma_{G}\right)= & \frac{n(n-1)}{2} \sqrt{\frac{\left(n^{2}-n+1\right)+\left(n^{2}-n+1\right)-2}{\left(n^{2}-n+1\right)\left(n^{2}-n+1\right)}} \\
& +n \sqrt{\frac{\left(n^{2}-n+1\right)+(2 n-1)-2}{\left(n^{2}-n+1\right)(2 n-1)}},
\end{aligned}
$$

$$
\begin{aligned}
\mathrm{ABC}_{4}\left(\Gamma_{G}\right)= & \frac{(n-2)(n-3) \sqrt{2 n^{2}}}{2\left(n^{2}+1\right)}+\frac{2(n-2)}{n+1} \sqrt{\frac{2 n^{2}+2 n}{n^{2}+1}} \\
& +\frac{\sqrt{2 n^{2}+4 n}}{(n+1)^{2}}+\frac{2 n}{n+1} \sqrt{\frac{n^{2}+6 n}{4 n+1}}+\frac{n \sqrt{8 n}}{2(4 n+1)}
\end{aligned}
$$

The required formulas for both the indices one can get by performing an easy simplification.

and for even values of $n$, we have 
Theorem 8. For $n \geq 3$, let $\Gamma_{G}$ be a commuting graph of $\Gamma=D_{n}$, then

$$
H_{r}\left(\Gamma_{G}\right)=\left\{\begin{array}{l}
\frac{3 n^{2}+2 n-4}{2(3 n-2)}, \\
\frac{(n-2)\left(3 n^{2}-3 n-2\right)}{2(n-1)(3 n-2)}+\frac{6(n+1)+n(2 n-1)(n+13)}{6(n+1)(2 n-1)} .
\end{array}\right.
$$

Proof. By applying the formula of the harmonic index, using the edge partition given in Table 3 , we have

$$
H_{r}\left(\Gamma_{G}\right)=\left\{\begin{array}{l}
\frac{(n-1)(n-2)}{2(n-1)}+\frac{2(n-1)}{3 n-2}+1, \\
\frac{(n-2)(n-3)}{2(n-1)}+\frac{4(n-2)}{3 n-2}+\frac{1}{2 n-1}+\frac{2 n}{n+1}+\frac{n}{6} .
\end{array}\right.
$$

Some simplifications yield the required values of the harmonic index.

\section{Topological Properties of Noncommuting Graph $G_{\Gamma}$}

In this section, we compute the reciprocal complementary Wiener, Harary, general Randić, $\mathrm{ABC}, \mathrm{ABC}_{4}, \mathrm{GA}, \mathrm{GA}_{5}$, and harmonic indices of $G_{\Gamma}$.
Theorem 9. For $n \geq 3$, let $G_{\Gamma}$ be a noncommuting graph of $\Gamma=D_{n}$, then

$$
\operatorname{RCW}\left(G_{\Gamma}\right)= \begin{cases}\frac{1}{12}\left(15 n^{2}-19 n+4\right), & \text { when } n \text { is odd, } \\ \frac{1}{12}\left(15 n^{2}-34 n+28\right), & \text { when } n \text { is even. }\end{cases}
$$

Proof. Since the diameter of $G_{\Gamma}$ is 2 , so by sing the vertex partition, given in Table 4, in formula (7) of the reciprocal complimentary Wiener index, we have

$$
\operatorname{RCW}\left(G_{\Gamma}\right)= \begin{cases}\frac{2 n-1}{3}+\frac{n(n-1)}{2}+\frac{(n-1)(3 n-4)}{4}, & \text { when } n \text { is odd, } \\ \frac{2 n-2}{3}+\frac{n(n-1)}{2}+\frac{3(n-2)(n-2)}{4}, & \text { when } n \text { is even. }\end{cases}
$$

Exact values for this index are due to some easy calculations.

Theorem 10. For $n \geq 3$, let $G_{\Gamma}$ be a noncommuting graph of $\Gamma=D_{n}$, then

$$
H\left(G_{\Gamma}\right)= \begin{cases}\frac{1}{4}\left(7 n^{2}-8 n+1\right), & \text { when } n \text { is odd, } \\ \frac{1}{4}\left(7 n^{2}-16 n+6\right), & \text { when } n \text { is even. }\end{cases}
$$

Proof. By sing the vertex partition, given in Table 4, in formula (9) of the Harary index, we have

$$
H\left(G_{\Gamma}\right)= \begin{cases}\frac{n(2 n-2)}{2}+\frac{(n-1)(3 n-1)}{4}, & \text { when } n \text { is odd } \\ \frac{n(4 n-7)}{4}+\frac{3(n-2)(n-1)}{4}, & \text { when } n \text { is even. }\end{cases}
$$

By performing some algebraic computations, one can obtain the required Harary index.

Theorem 11. For $n \geq 3$, let $G_{\Gamma}$ be a noncommuting graph of $\Gamma=D_{n}$. Then, for odd values of $n$, 


$$
R_{\alpha}\left(G_{\Gamma}\right)= \begin{cases}2 n(n-1)^{2}(2 n-1), & \text { for } \alpha=1, \\ \frac{5 n-4}{8(n-1)}, & \text { for } \alpha=-1, \\ n(n-1) \sqrt{2 n(n-1)}+n-1, & \text { for } \alpha=\frac{1}{2}, \\ \sqrt{\frac{n(n-1)}{2}+\frac{n}{4},} & \text { for } \alpha=-\frac{1}{2},\end{cases}
$$

Proof. Using the edge partition, given in Table 5, in the formula of general Randic index $R_{\alpha}$ for $\alpha=1,-1,1 / 2,-(1 / 2)$, we have

and for even values of $n$,

$$
R_{\alpha}\left(G_{\Gamma}\right)= \begin{cases}4 n(n-2)^{2}(n-1), & \text { for } \alpha=1, \\ \frac{10 n-16}{16 n-32}, & \text { for } \alpha=-1, \\ n(n-2) \sqrt{n(2 n-4)}+n(n-2)^{2}, & \text { for } \alpha=\frac{1}{2}, \\ \frac{4 n(n-2)+n \sqrt{2 n(n-2)}}{4 \sqrt{2 n(n-2)}}, & \text { for } \alpha=-\frac{1}{2} .\end{cases}
$$

$$
\begin{aligned}
& R_{1}\left(G_{\Gamma}\right)= \begin{cases}n^{2}(n-1)(2 n-2)+\frac{n(n-1)(2 n-2)^{2}}{2}, & \text { when } n \text { is odd, } \\
n^{2}(n-2)(2 n-4)+\frac{n(n-2)(2 n-4)^{2}}{2}, & \text { when } n \text { is even, }\end{cases} \\
& R_{-1}\left(G_{\Gamma}\right)= \begin{cases}\frac{n(n-1)}{n(2 n-2)}+\frac{n(n-1)}{2(2 n-2)^{2}}, & \text { when } n \text { is odd } \\
\frac{n(n-2)}{n(2 n-4)}+\frac{n(n-2)}{2(2 n-4)^{2}}, & \text { when } n \text { is even, }\end{cases} \\
& R_{1 / 2}\left(G_{\Gamma}\right)= \begin{cases}n(n-1) \sqrt{n(2 n-2)}+\frac{n(n-1)(2 n-2)}{2}, & \text { when } n \text { is odd, } \\
n(n-2) \sqrt{n(2 n-4)}+\frac{n(n-2)(2 n-4)}{2}, & \text { when } n \text { is even, }\end{cases} \\
& R_{-(1 / 2)}\left(G_{\Gamma}\right)= \begin{cases}\frac{n(n-1)}{\sqrt{n(2 n-2)}}+\frac{n(n-1)}{2(2 n-2)}, & \text { when } n \text { is odd }, \\
\frac{n(n-2)}{\sqrt{n(2 n-4)}}+\frac{n(n-2)}{2(2 n-4)}, & \text { when } n \text { is even. }\end{cases}
\end{aligned}
$$
results.

By performing some simplifications, we get the required
Theorem 12. Let $G_{\Gamma}$ be a noncommuting graph of $\Gamma=D_{n}$, for $n \geq 3$, then 


$$
\begin{aligned}
& \mathrm{GA}\left(G_{\Gamma}\right)= \begin{cases}\frac{2(2 n(n-1))^{3 / 2}+n(n-1)(3 n-2)}{2(3 n-2)}, & \text { when } n \text { is odd, } \\
\frac{2(2 n(n-2))^{3 / 2}+n(n-2)(3 n-4)}{2(3 n-4)}, & \text { when } n \text { is even, }\end{cases} \\
& \mathrm{GA}_{5}\left(G_{\Gamma}\right)= \begin{cases}\frac{4 n \sqrt{2 n(n-1)^{2}(3 n-2)}+n(n-1)(5 n-2)}{10 n-4}, & \text { when } n \text { is odd, } \\
\frac{n(n-2)(5 n-4+\sqrt{32 n(3 n-4)})}{10 n-8}, & \text { when } n \text { is even. }\end{cases}
\end{aligned}
$$

Proof. Applying formulas of the geometric arithmetic index and its fifth version, using the edge partition given in Table 5 , we have

$$
\begin{aligned}
& \mathrm{GA}\left(G_{\Gamma}\right)=\left\{\begin{array}{l}
\frac{2 n(n-1) \sqrt{n(2 n-2)}}{3 n-2}+\frac{n(n-1)}{2}, \quad \text { when } n \text { is odd, } \\
\frac{2 n(n-2) \sqrt{n(2 n-4)}+\frac{n(n-2)}{3 n-4},}{2} \text { when } n \text { is even, } \\
\mathrm{GA}_{5}\left(G_{\Gamma}\right)=\left\{\begin{array}{l}
\frac{2 n(n-1) \sqrt{2 n(n-1)^{2}(3 n-2)}}{2 n(n-1)+(n-1)(3 n-2)}+\frac{n(n-1)}{2}, \\
\frac{2 n(n-2) \sqrt{2 n(n-2)^{2}(3 n-4)}}{2 n(n-2)+(n-2)(3 n-4)}+\frac{n(n-2)}{2},
\end{array} \text { when } n\right. \text { is odd, }
\end{array}\right.
\end{aligned}
$$

The required values of the geometric arithmetic index and its fifth version can be obtained after some simplifications.
Theorem 13. For $n \geq 3$, let $G_{\Gamma}$ be a noncommuting graph of $\Gamma=D_{n}$, then

$$
\begin{aligned}
& \mathrm{ABC}\left(G_{\Gamma}\right)= \begin{cases}\sqrt{\frac{n(n-1)(3 n-4)}{2}}+\sqrt{\frac{n^{2}(2 n-3)}{8}}, & \text { when } n \text { is odd, } \\
\sqrt{\frac{n(n-2)(3 n-6)}{2}}+\frac{n \sqrt{4 n-10}}{4}, & \text { when } n \text { is even, }\end{cases} \\
& \operatorname{ABC}_{4}\left(G_{\Gamma}\right)= \begin{cases}\sqrt{\frac{n^{2}(n-1)^{2}\left(5 n^{2}-7 n\right)}{6 n^{4}-16 n^{3}+14 n^{2}-4 n}+\frac{\sqrt{n^{2}\left(6 n^{2}-10 n+2\right)}}{2(3 n-2)},}, & \text { when } n \text { is odd, } \\
\sqrt{\frac{5 n^{3}-14 n^{2}+6 n}{3 n-4}}+\frac{\sqrt{2 n^{2}(n-1)(3 n-7)}}{2(3 n-4)}, & \text { when } n \text { is even. }\end{cases}
\end{aligned}
$$

Proof. By using the edge partition, given in Table 5, in formulas of $\mathrm{ABC}$ and $\mathrm{ABC}_{4}$ indices, we have 


$$
\operatorname{ABC}\left(G_{\Gamma}\right)= \begin{cases}n(n-1) \sqrt{\frac{3 n-4}{2 n(n-1)}+\frac{n(n-1) \sqrt{4 n-6}}{2(2 n-2)},} & \text { when } n \text { is odd, } \\ n(n-2) \sqrt{\frac{3 n-6}{n(2 n-4)}+\frac{n(n-2) \sqrt{4 n-10}}{2(2 n-4)},} & \text { when } n \text { is even. }\end{cases}
$$

Also, for odd values of $n$, we have

$$
\begin{aligned}
\operatorname{ABC}_{4}\left(G_{\Gamma}\right) & =n(n-1) \sqrt{\frac{2 n(n-1)+(n-1)(3 n-2)-2}{2 n(n-1)^{2}(3 n-2)}} \\
& +\frac{n(n-1)}{2} \sqrt{\frac{2(n-1)(3 n-2)-2}{((n-1)(3 n-2))^{2}}}
\end{aligned}
$$

and for even values of $n$, we have

$$
\begin{aligned}
\mathrm{ABC}_{4}\left(G_{\Gamma}\right)= & n(n-2) \sqrt{\frac{2 n(n-2)+(n-2)(3 n-4)-2}{2 n(n-2)^{2}(3 n-4)}} \\
& +\frac{n(n-2) \sqrt{2(n-2)(3 n-4)-2}}{2(n-2)(3 n-4)} .
\end{aligned}
$$

The required formulas for both the indices one can get by performing an easy simplification.

Theorem 14. For $n \geq 3$, let $G_{\Gamma}$ be a noncommuting graph of $\Gamma=D_{n}$, then

$$
H_{r}\left(G_{\Gamma}\right)= \begin{cases}\frac{n(11 n-10)}{4(3 n-2)}, & \text { when } n \text { is odd, } \\ \frac{n(11 n-20)}{4(3 n-4)}, & \text { when } n \text { is even. }\end{cases}
$$

Proof. By applying the formula of the harmonic index, using the edge partition given in Table 5, we have

$$
H_{r}\left(G_{\Gamma}\right)= \begin{cases}\frac{2 n(n-1)}{3 n-2}+\frac{n(n-1)}{2(2 n-2)}, & \text { when } n \text { is odd, } \\ \frac{2 n(n-2)}{3 n-4}+\frac{n(n-2)}{2(2 n-4)}, & \text { when } n \text { is even. }\end{cases}
$$

Some simplifications yield the required values of the harmonic index.

\section{Concluding Remarks}

An algebraic structure plays a vital role in chemistry to form chemical compound structures and in investigating various chemical properties of chemical compounds in these structures. Here, we considered a very well-known algebraic structure, called the group of symmetries of regular gons (the dihedral group), which has remarkable contribution in the theory of electron structures and molecular vibrations. We considered one algebraic property, namely, commutation property, on the dihedral group and associated two graphs (chemical structure) with the group of symmetries. We computed some distance-based and degree-based topological properties of these associated graphs by computing the exact formulae of the Wiener index, reciprocal complementary Wiener index, Schultz molecular topological index, Harary index, Randić index, geometric arithmetic indices, atomic bond connectivity indices, and harmonic index. All the indices are numeric quantities and, in fact, this work is a theoretical contribution in the theory of topological indices with the unique algebraic structure, and it can be very helpful to predict the bioactivity of chemical compounds using physicochemical properties in QSAR/QSPR studied.

\section{Data Availability}

The data used to support the findings of this study are included within the article.

\section{Conflicts of Interest}

The authors declare that they have no conflicts of interest.

\section{Acknowledgments}

This work was supported by Top-Notch Talents Cultivation Project of Anhui Higher Education (Grant no. gxyq2017081) and Natural Science Fund of Education Department of Anhui Province (Grant no. KJ2017A4691).

\section{References}

[1] M. Mirzargar and A. R. Ashrafi, "Some distance-based topological indices of a non-commuting graph," Hacettepe Journal of Mathematics and Statistics, vol. 41, no. 4, pp. 515-526, 2012.

[2] H. Wiener, "Structural determination of paraffin boiling points," Journal of the American Chemical Society, vol. 69, no. 1, pp. 17-20, 1947.

[3] G. Caporossi, I. Gutman, P. Hansen, and L. Pavlović, "Graphs with maximum connectivity index," Computational Biology and Chemistry, vol. 27, no. 1, pp. 85-90, 2003.

[4] A. Graovac, M. Ghorbani, and M. A. Hosseinzadeh, "Computing fifth goemetric-arithmetic index for nanostar dendrimers," Journal of Mathematical Nanoscience, vol. 1, pp. 33-42, 2011. 
[5] M. Ghorbani and M. A. Hosseinzadeh, "Computing $\mathrm{ABC}_{4}$ index of nanostar dendrimers," Advanced Materials-Rapid Communications, vol. 4, pp. 1419-1422, 2010.

[6] I. Gutman, "Degree-based topological indices," Croatica Chemica Acta, vol. 86, no. 4, pp. 351-361, 2013.

[7] S. Hayat and M. Imran, "Computation of topological indices of certain networks," Applied Mathematics and Computation, vol. 240, pp. 213-228, 2014.

[8] M. Imran, S. Hayat, and M. Y. H. Mailk, "On topological indices of certain interconnection networks," Applied Mathematics and Computation, vol. 244, pp. 936-951, 2014.

[9] K. Xu, M. Liu, K. C. Das, I. Gutman, and B. Furtula, “A survey on graphs extremal with respect to distance-based topological indices," MATCH Communications in Mathematical and in Computer Chemistry, vol. 71, pp. 461-508, 2014.

[10] S. Hayat, M. Imran, and J.-B. Liu, "An efficient computational technique for degree and distance based topological descriptors with applications," IEEE Access, vol. 7, pp. 3227632296, 2019.

[11] S. Hayat, S. Wang, and J.-B. Liu, "Valency-based topological descriptors of chemical networks and their applications," Applied Mathematical Modelling, vol. 60, pp. 164-178, 2018.

[12] S. Hayat, "Computing distance-based topological descriptors of complex chemical networks: new theoretical techniques," Chemical Physics Letters, vol. 688, pp. 51-58, 2017.

[13] J.-B. Liu, C. Wang, S. Wang, and B. Wei, "Zagreb indices and multiplicative Zagreb indices of Eulerian graphs," Bulletin of the Malaysian Mathematical Sciences Society, vol. 42, no. 1, pp. 67-78, 2019.

[14] J.-B. Liu and X.-F. Pan, "Minimizing Kirchhoff index among graphs with a given vertex bipartiteness," Applied Mathematics and Computation, vol. 291, pp. 84-88, 2016.

[15] J.-B. Liu, X.-F. Pan, F.-T. Hu, and F.-F. Hu, "Asymptotic Laplacian-energy-like invariant of lattices," Applied Mathematics and Computation, vol. 253, pp. 205-214, 2015.

[16] F. Ali, M. Salman, and S. Huang, "On the commuting graph of dihedral group," Communications in Algebra, vol. 44, no. 6, pp. 2389-2401, 2016.

[17] D. Bunday, "The connectivity of commuting graphs," Journal of Combinatorial Theory, Series A, vol. 113, no. 6, pp. 995$1007,2006$.

[18] B. H. Neumann, "A problem of Paul Erdös on groups," Journal of the Australian Mathematical Society, vol. 21, no. 4, pp. 467-472, 1976.

[19] A. Abdollahi, S. Akbari, and H. R. Maimani, "Non-commuting graph of a group," Journal of Algebra, vol. 298, no. 2, pp. 468-492, 2006.

[20] A. R. Moghaddamfar, W. J. Shi, W. Zhou, and A. R. Zokayi, "On the noncommuting graph associated with a finite group," Siberian Mathematical Journal, vol. 46, no. 2, pp. 325-332, 2005.

[21] O. Ivancivc, "QSAR comparative study of Wiener descriptors for weighted molecular graphs," Journal of Chemical Information and Computer Sciences, vol. 40, no. 6, pp. 1412-1422, 2000.

[22] H. P. Schultz, "Topological organic chemistry. 1. graph theory and topological indices of alkanes," Journal of Chemical Information and Modeling, vol. 29, no. 3, pp. 227-228, 1989.

[23] O. Ivancivc, T. S. Balaban, and A. T. Balaban, "Design of topological indices. Part 4. Reciprocal distance matrix, related local vertex invariants and topological indices," Journal of Mathematical Chemistry, vol. 12, no. 1, pp. 309-318, 1993.

[24] D. Plavšić, S. Nikolić, N. Trinajstić, and Z. Mihalić, "On the Harary index for the characterization of chemical graphs,"
Journal of Mathematical Chemistry, vol. 12, no. 1, pp. 235-250, 1993.

[25] M. Randic, "Characterization of molecular branching," Journal of the American Chemical Society, vol. 97, no. 23, pp. 6609-6615, 1975.

[26] D. Amić, D. Bešlo, B. Lučić, S. Nikolić, and N. Trinajstić, "The vertex-connectivity index revisited," Journal of Chemical Information and Computer Sciences, vol. 38, no. 5, pp. 819822, 1998.

[27] B. Bollobás and P. Erdös, "Graph of extremal weights," Ars Combinatoria, vol. 50, pp. 225-233, 1998.

[28] D. Vukičević and B. Furtula, "Topological index based on the ratios of geometrical and arithmetical means of end-vertex degrees of edges," Journal of Mathematical Chemistry, vol. 46, no. 4, pp. 1369-1376, 2009.

[29] E. Estrada, L. Torres, L. Rodríguez, and I. Gutman, “An atombond connectivity index: modelling the enthalpy of formation of alkanes," Indian Journal of Chemistry, vol. 37, no. 10, pp. 849-855, 1998.

[30] S. Fajtlowicz, "On conjectures of Grafitti II," Congress Numer, vol. 60, pp. 189-197, 1987. 